\title{
THE FRACTAL STRUCTURE OF LIMIT SET OF SOLUTION SPACE OF A DOUBLY PERIODIC RICATTI EQUATION
}

\author{
KE-YING GUAN \\ Department of Applied Mathematics and Physics \\ Beijing University of Aeronautics and Astronautics \\ Beijing 100083, P R CHINA
}

(Received December 26, 1995)

\begin{abstract}
The limit set of the Kleinian group of a given doubly periodic Riccati equation is proved to have a fractal structure if the parameter $\delta(\lambda)$ of the equation is greater than $3+2 \sqrt{2}$, and a possible Hausdorff dimension is suggested to the limit set
\end{abstract}

KEY WORDS AND PHRASES: Kleinian group of a Riccati equation, limit set, fractal 1991 AMS SUBJECT CLASSIFICATION CODES: $34 \mathrm{C} 35$

It is well known that the limit sets of many nonlinear autonomous systems have fractal structures (see [1][2][3]) However, it is very difficult to verify the fractal structure analytically and exactly for a given system, since the nonlinear autonomous system is usually not integrable by traditional quadratures In [4] and [5] the periodic solutions and the structures of Riemann surfaces of solutions to a doubly periodic Riccati equation are discussed, and the monodromy group (a Kleinian group (see [6])) of the equation is established concretely for the study of the structure of the limit set of the solution space, and it is conjectured that the limit set has a fractal structure under certain conditions In this paper, this conjecture will be proved by means of the Kleinian group Some relevant conceptions and known results will be introduced at first for convenience

Consider the Riccati equation

$$
\frac{d z}{d t}=z^{2}(t)+\lambda \varrho(t)
$$

where

$$
t \in C, \quad z \in \widehat{C}(\widehat{C}=C \bigcup\{\infty\}), \quad \lambda \in R^{+},
$$

and $\varrho(t)$ is a Weierstrass elliptic function satisfying the following equation

$$
\left[\varrho^{\prime}(t)\right]^{2}=4\left[\varrho^{2}(t)-1\right] \varrho(t)
$$

with the initial condition $\varrho(0)=0$. $\varrho(t)$ has two periods

and

$$
\omega_{1}=\Gamma\left(\frac{1}{2}\right) \Gamma\left(\frac{1}{4}\right) / 2 \Gamma\left(\frac{3}{4}\right)=2 \alpha
$$

$$
\omega_{2}=2 \alpha i
$$

As a global analytic function (see [7]), a solution of equation (1) is usually multiple-valued It is proved in [5] that (1) has two particular solutions $z_{r_{1}}(t)$ and $z_{r_{2}}(t)$, which have respectively single-valued and analytical periodic branches $\bar{z}_{r_{1}}(t)$ and $\bar{z}_{r_{2}}(t)$ with the period $2 \alpha$ in the strip region

$$
D=\{t|| \operatorname{Im}(t) \mid<\alpha\},
$$


and that (1) has two other particular solutions $z_{\imath_{1}}(t)$ and $z_{\imath_{2}}(t)$, which have respectively single-valued and analytical periodic branches $\bar{z}_{i_{\mathrm{i}}}(t)$ and $\bar{z}_{z_{2}}(t)$ with the period $2 \alpha i$ in the strip region

$$
D^{\prime}=\{t|| \operatorname{Re}(t) \mid<\alpha\} .
$$

Since $\varrho(t)$ is doubly periodic, equation (1) can be rewritten as an autonomous system

$$
\left\{\begin{array}{l}
\frac{d z}{d t}=z^{2}+\lambda \varrho(\sigma), \\
\frac{d \sigma}{d t}=1, \sigma(0)=0,
\end{array}\right.
$$

where

$$
(z, \sigma) \in \widehat{C} \times T_{0}^{2}, \quad t \in C,
$$

and $T_{0}^{2}$ is the two dimensional torus with a point like hole $P_{0}$ corresponding to the pole of $\varrho(t)$ at

$$
t=(2 m+1) \alpha+(2 n+1) \alpha i, \quad(m, n) \in Z^{2} .
$$

Let $z_{l o c}(\sigma)$ represent a local solution of (2) defined in the open region

$$
\{\sigma \mid \operatorname{Re}(\sigma) \in(-\alpha, \alpha), \operatorname{Im}(\sigma) \in(-\alpha, \alpha)\},
$$

with the initial value $z_{l o c}(0)$

A global solution $z_{\text {glob }}(\sigma)$ of system (2) is still mutiple-valued. The Riemann surface of $z_{\text {glob }}(\sigma)$ can be treated as the phase surface of the global solution imbedded in $\widehat{C} \times T_{0}^{2}$ So $z_{\text {glob }}(0)$, which is a set of infinitely many local values $z_{l o c}(0) \in \widehat{C}$, is corresponding to the intersection set between the Riemann surface of $z_{g l o b}(\sigma)$ and the phase plane $\sigma=0$ For convenience, we introduce the transformation

$$
c=\frac{z_{l o c}(0)-\bar{z}_{r_{1}(0)}}{z_{l o c}(0)-\bar{z}_{r_{2}}(0)}
$$

which transforms the initial value $z_{l o c}(0)$ into the value $c$. Therefore every value $c \in \widehat{C}$ is corresponding to a definite local solution $z_{l o c}(\sigma)$ of the initial value $z_{l o c}(0)$. This correspondence reflects some important topological properties of the solution space of (2) to the extended $\widehat{C}$ plane For instance, if the local solution sequence $\left\{z_{l o c, n}(\sigma)\right\}$ converges to a local solution $z_{l o c, 0}(\sigma)$, then their corresponding $c$ value sequence $\left\{c_{n}\right\}$ converges to the $c$-value $c_{0}$ of $z_{l o c, 0}(\sigma)$, and vice versa. The monodromy group of the system (2) - a Kleinian group $G$ (see [6]) has two generators $E$ and $U \quad E$ is corresponding to an analytical continuation transformation, which continues a local solution corresponding to any given $c$ value $c \in \widehat{C}$ along the direction of the latitude of $T_{0}^{2}$ (or the direction of the real axis of the $C$-plane) for a lap to the new local solution corresponding to the $c$-value $E(c)$, and $U$ 'is corresponding to the transformation, which continues analytically a local solution of the $c$-value $c \in \widehat{C}$ along the direction of the longitude of $T_{0}^{2}$ (or the direction of the imaginary axis of the $C$-plane) for a lap to the new local solution of the $c$-value $U(c)$ In [5] we obtained that

where

$$
\left\{\begin{array}{l}
E(C)=\delta c \\
U(c)=\frac{\imath(\delta-1)+(\delta+1) c}{(\delta+1)-\imath(\delta-1) c}
\end{array}\right.
$$

$$
\delta=\delta(\lambda)=\exp \int_{0}^{2 \alpha}\left[\bar{z}_{r_{1}}(t)-\bar{z}_{r_{2}}(t)\right] d t
$$

Since $\lambda \in R^{+}, \bar{z}_{r_{1}}(t)>\bar{z}_{r_{2}}(t)$ for all $t \in R$, and therefore, $\delta(\lambda) \in(1,+\infty)$ Based on the result in [8], it can be proved that $\delta(\lambda)$ is a continuous and monotone increasing function in $\lambda$ and that

$$
\left\{\begin{array}{l}
\lim _{\lambda \rightarrow 0_{+}} \delta(\lambda)=1 \\
\lim _{\lambda \rightarrow+\infty} \delta(\lambda)=+\infty
\end{array}\right.
$$

An element of the group $G$ can be represented as

$$
g=U^{n_{k}} E^{m_{k}} U^{n_{k-1}} E^{m_{k-1}} \ldots U^{n_{1}} E^{m_{1}},
$$


where $m_{2}, n_{2} \in Z$ for $i=1,2, \ldots, k$ Clearly, $g$ is a Mobius transformation on $\widehat{C}$ Let

$$
\begin{aligned}
C^{+} & =\{c \mid c \in \widehat{C}, \operatorname{Im}(c)>0\}, \\
C^{-} & =\{c \mid c \in \widehat{C}, \operatorname{Im}(c)<0\}, \\
C_{\imath} & =\widehat{C} \backslash\left[C^{+} \bigcup C^{-}\right] .
\end{aligned}
$$

In fact, $C_{2}$ is the extended imaginary axis It is easy to check that for any $g \in G$,

$$
g\left(C^{+}\right)=C^{+}, \quad g\left(C^{-}\right)=C^{-}, \quad g\left(C_{\imath}\right)=C_{\imath} .
$$

Therefore, $C^{+}, C^{-}$and $C_{\imath}$ are $G$-invariant

Assume that $c_{0}$ is the $c$-value of a given local solution $z_{l o c, 0}(\sigma)$, then the set of the $c$-values of all the local solutions obtained from $z_{l o c, 0}(\sigma)$ by continuation can be represented as

$$
S\left(c_{0}\right)=\left\{g\left(c_{0}\right) \mid g \in G\right\} .
$$

The $c$-values of the local periodic solutions $\bar{z}_{r_{1}}(t), \bar{z}_{r_{2}}(t), \bar{z}_{z_{1}}(t)$ and $\bar{z}_{\imath_{2}}(t)$ are respectively $0, \infty, i$ and $-i$ (see [5]) For given $\lambda \in R^{+}$, let $V(\lambda)$ be the union of sets

$$
V(\lambda)=S(0) \bigcup S(\infty) \bigcup S(i) \bigcup S(-i)
$$

The following is the main theorem of this paper.

THEOREM. If

$$
\delta(\lambda)>3+2 \sqrt{2}
$$

(the numerical work shows that $\delta(0.227) \approx 3+2 \sqrt{2}$ ), then $\overline{V(\lambda)}$, which is the limit set of the Kleinian group $G$ of the system (2), is a totally disconnected (or thin) and perfect subset of $C_{2}$, or in other words, $G$ is a non-elementary Fuchsian group of the second kind (ref [9]), and according to a consideration on similarities, its Hausdorff dimension can be evaluated as

$$
D_{f}(\overline{V(\lambda)}) \approx \frac{\ln 3}{\ln \delta(\lambda)}
$$

The proof of this theorem can be completed by verifying the following facts and propositions

FACT 1. $C_{\imath}$ is a one-dimensional manifold homeomorphic to the unit circle There exist two kinds of natural symmetries

(i) if $x, y \in C_{2}$ and $x=-y$, then they are said to be symmetric to each other with respect to the points 0 and $\infty$;

(ii) if $x, y \in C_{\imath}$ and $\operatorname{Im}(x)=\frac{1}{\operatorname{Im}(y)}$, then they are said to be symmetric to each other with respect to the points $i$ and $-i$

Obviously, $i$ and $-i$ are symmetric with respect to 0 and $\infty$, and 0 and $\infty$ are symmetric with respect to $i$ and $-i$ (see Figure 1).

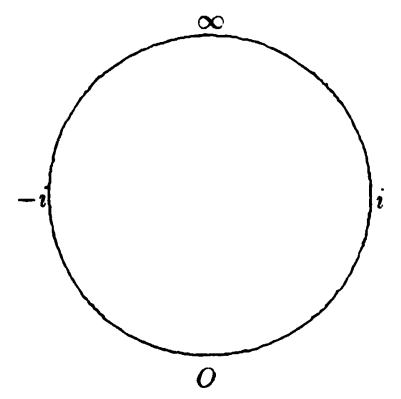

Figure 1. $C_{\imath}$ and its natural symmetries. 
PROPOSITION 1. On the subset $C_{\imath}$, the transform $E$ preserves the symmetry with respect to 0 and $\infty$, i e, $E(x)$ and $E(y)$ are symmetric with respect to 0 and $\infty$ if $x$ and $y$ are symmetric with respect to 0 and $\infty$, and the transformation $U$ preserves the symmetry with respect to $i$ and $-i$

PROPOSITION 2. When

$$
\delta(\lambda)>3+2 \sqrt{2}
$$

if let

$$
\begin{aligned}
& \alpha=\frac{1}{2 \delta}\left[(\delta-1)-\sqrt{(\delta-1)^{2}-4 \delta}\right], \\
& \beta=\frac{1}{2 \delta}\left[(\delta-1)+\sqrt{(\delta-1)^{2}-4 \delta}\right],
\end{aligned}
$$

then $0<\alpha<\beta<1$, and under the action of any $g \in G$, on $C_{2}$, the points,

and the open interval,

$$
u_{1}=\alpha i, \quad u_{2}=\beta i
$$

$$
I_{0}=\{\gamma i \mid \gamma \in(\alpha, \beta)\}
$$

are changed respectively into the points $g\left(u_{1}\right)$ and $g\left(u_{2}\right)$ and into the open interval $g\left(I_{0}\right)$ between $g\left(u_{1}\right)$ and $g\left(u_{2}\right)$ Especially,

$$
\begin{aligned}
E\left(u_{1}\right) & =\delta u_{1}=\frac{1}{\beta} i, & E\left(u_{2}\right) & =\delta u_{2}=\frac{1}{\alpha} i \\
U^{-1}\left(u_{1}\right) & =-u_{2}, & U^{-1}\left(u_{2}\right) & =-u_{1}, \\
U^{-1}\left(E\left(u_{1}\right)\right) & =-E\left(u_{2}\right), & U^{-1}\left(E\left(u_{2}\right)\right) & =-E\left(u_{1}\right) .
\end{aligned}
$$

And the intervals $I_{0}$ and $U^{-1}\left(I_{0}\right)$ are symmetric with respect to 0 and $\infty$, the intervals $E\left(I_{0}\right)$ and $U^{-1}\left(E\left(I_{0}\right)\right)$ are symmetric with respect to 0 and $\infty$, the intervals $I_{0}$ and $E\left(I_{0}\right)$ are symmetric with respect to $i$ and $-i$, and the intervals $U^{-1}\left(I_{0}\right)$ and $U^{-1}\left(E\left(I_{0}\right)\right)$ are symmetric with respect to $\imath$ and $-\imath$

Let

$$
\begin{gathered}
O=I_{0} \bigcup E\left(I_{0}\right) \bigcup U^{-1}\left(E\left(I_{0}\right)\right), \\
F=C_{\imath} \backslash O .
\end{gathered}
$$

FACT 2. The closed set $F$ is formed with four separated closed intervals $F_{0}, F_{\infty}, F_{\imath}$ and $F_{-\imath}$, and the points $0, \infty, i$ and $-i$ are located on them as their symmetric centers respectively (see Figure 2)

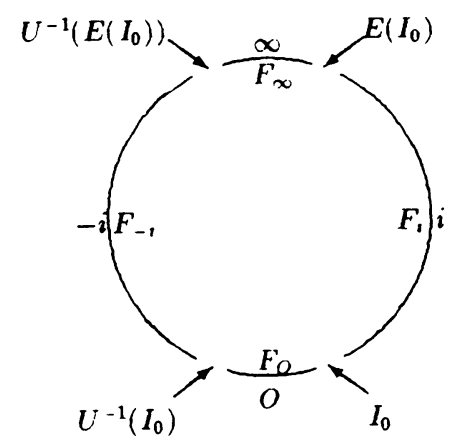

Figure 2. The open intervals $I_{0}, E\left(I_{0}\right), U^{-1}\left(I_{0}\right), U^{-1}\left(E\left(I_{0}\right)\right)$, and the closed intervals $F_{O}, F_{\infty}, F_{2}, F_{-2}(\delta=8)$ 


\section{PROPOSITION 3.}

$$
\begin{gathered}
E\left(F_{0}\right)=F_{-\imath} \bigcup U^{-1}\left(I_{0}\right) \bigcup F_{0} \bigcup I_{0} \bigcup F_{\imath}, \\
E^{-1}\left(F_{\infty}\right)=F_{-\imath} \bigcup U^{-1}\left(E\left(I_{0}\right)\right) \bigcup F_{\infty} \bigcup E\left(I_{0}\right) \bigcup F_{\imath}, \\
U\left(F_{0} \bigcup I_{0} \bigcup F_{\imath} \bigcup E\left(I_{0}\right) \bigcup F_{\infty}\right)=F_{\imath}
\end{gathered}
$$

and

$$
U^{-1}\left(F_{0} \bigcup U^{-1}\left(I_{0}\right) \bigcup F_{-\imath} \bigcup U^{-1}\left(E\left(I_{0}\right)\right) \bigcup F_{\infty}\right)=F_{-\imath}
$$

Let

$$
F_{ \pm \imath}=F_{\imath} \bigcup F_{-\imath}, \quad F_{0, \infty}=F_{0} \bigcup F_{\infty} .
$$

FACT 3. The family of transformations $\left\{E^{m}\right\}_{m \in Z_{0}}$ (note $\mathbb{Z}_{0}$ is the set of non-zero integers) change $F_{ \pm 2}$ to a family of separated closed intervals in $F_{0, \infty}$, and 0 and $\infty$ are the accumulation points of these intervals The family of transformation $\left\{U^{m}\right\}_{m \in \mathbf{Z}_{0}}$ change $F_{0, \infty}$ to a family of separated closed intervals in $F_{ \pm \imath}$, and $i$ and $-i$ are the accumulation points of these intervals

Let

$$
\begin{aligned}
& F_{0, \infty}(1)=\bigcup_{m \in \mathbf{Z}_{0}} E^{m}\left(F_{ \pm \imath}\right), \\
& F_{ \pm \imath}(1)=\bigcup_{m \in \mathbf{Z}_{0}} U^{m}\left(F_{0, \infty}\right) .
\end{aligned}
$$

And for any positive integer $n>1$, we may define inductively that

$$
F_{0, \infty}(n)=\bigcup_{m \in \mathbb{Z}_{0}} E^{m}\left(\overline{F_{ \pm \imath}(n-1)}\right),
$$

and that

$$
F_{ \pm 2}(n)=\bigcup_{m \in \mathbf{Z}_{0}} U^{m}\left(\overline{F_{0, \infty}(n-1)}\right) .
$$

FACT 4. The closed set $\overline{F_{0, \infty}(n)} \bigcup \overline{F_{ \pm \imath}(n)}$ is formed with a series of separated closed intervals and their accumulation points. The endpoints of these intervals belong to

$$
S\left(u_{1}\right) \bigcup S\left(u_{2}\right)
$$

and these accumulation points belong to

$$
S(0) \bigcup S(\infty) \bigcup S(i) \bigcup S(-i)
$$

Let $P(n)$ denote the set of the endpoints of these closed intervals and their accumulation points mentioned in Fact 4.

PROPOSITION 4. For any $n \in N$,

$$
\begin{gathered}
{\left[\overline{F_{0 . \infty}(n)} \bigcup \overline{F_{ \pm \imath}(n)}\right] \supset\left[\overline{F_{0 . \infty}(n+1)} \bigcup \overline{F_{ \pm \imath}(n+1)}\right],} \\
P(n) \subset P(n+1) .
\end{gathered}
$$

PROPOSITION 5. The set $\overline{V(\lambda)}$, just like the Cantor set, is constructed as the intersection of the monotone sequence of closed sets

$$
\left.\overline{V(\lambda)}=\bigcap_{n \in N} \overline{F_{0, \infty}(n)} \bigcup \overline{F_{ \pm 2}(n)}\right]
$$


and is a totally disconnected (or thin) and perfect subset in $C_{\imath}$ And the subsets $S(0), S(\infty), S(i)$, $S(-i), S\left(u_{1}\right)$ and $S\left(u_{2}\right)$ are dense in $\overline{V(\lambda)}$

PROPOSITION 6. $\overline{V(\lambda)}$ is the smallest non-empty $G$-invariant closed subset of $\widehat{C}$, and therefore it is the limit set of the Kleinian group $G$, and $G$ is a non-elementary Fuchsian group of the second kind (ref [9])

FACT 5. From the construction of the set $V(\lambda)$ we see that it has the following self-similar laws

(i) The structure of $\overline{V(\lambda)} \cap F_{0}$ is similar to the structure of $\overline{V(\lambda)} \cap F_{-2}$, and also to the structure of $\overline{V(\lambda)} \cap F_{2}$,

(ii) $E\left(\overline{V(\lambda)} \cap F_{0}\right)=\left[\overline{V(\lambda)} \cap F_{-2}\right] \cup\left[\overline{V(\lambda)} \cap F_{0}\right] \cup\left[\overline{V(\lambda)} \cap F_{2}\right]$

Notice that the length of $E\left(F_{0}\right)$ is $\delta$ times the length of $F_{0}$, and the length of $F_{-2}$ or of $F_{2}$ is different to the length of $F_{0}$ So the self-similar laws of $\overline{V(\lambda)}$ are a little complicated, and consequently, we cannot define and calculate strictly its Hausdorff dimension according to the method used commonly However, in loosening some requirements, we may have the following estimation

PROPOSITION 7. If the length difference between $F_{ \pm 2}$ and $F_{0}$ is neglected, the Hausdorff dimension of $\overline{V(\lambda)}$ can be evaluated as

$$
D_{f}(\overline{V(\lambda)}) \approx \frac{\ln 3}{\ln \delta(\lambda)} .
$$

This completes the proof of the main theorem of this paper The structure of the set $\overline{V(\lambda)}$ reflects the complexity of the limit set of the solution space of system (2) If the parameter

$$
\delta(\lambda) \in(1,3+2 \sqrt{2})
$$

then the open interval $I_{0}$ in Proposition 2 does not exist In this case, we may guess that $\overline{V(\lambda)}=C_{\imath}$, i e, $G$ is a Fuchsian group of the first kind (ref [9]) Our numerical results seem to support the main theorem and the conjecture

The fractal structure of the limit set of solution space is a kind of characters of non-integrable equation systems. In fact, according to the results in [10], if the limit set of an autonomous system has a fractal structures, then this system does not admit any nontrivial analytical Lie group in a relative large region In this sense, this system is not integrable

ACKNOWLEDGMENT. This work was supported by the National Natural Science Foundation of China.

\section{REFERENCES}

[1] THOMPSON, J.M T and STEWART, H.B., Nonlinear Dynamics and Chaos, John Wiley and Sons (1986).

[2] HOLDEN, A V., Chaos, Manchester University Press (1986)

[3] HUBBARD, J.H and WEST, B.H, Differential Equations, A Dynamical Systems Approach, Part I, Springer-Verlag (1991).

[4] GUAN, K.Y. and CHEN, Z.M., Doubly periodic solutions of the Ricatti equation in complex domain, Chinese Scrence Bullettn, 36, 23 (1991), 1943-1947.

[5] GUAN, K.Y. and CHEN, Z.M., On the complexity of the limit set of solution space of a doubly periodic Riccati equation, Science in China (Series A), 24, 8 (1994), 800-807

[6] ARNOLD, V.I. and IL'YASHENKO, Y.S., Ordinary Differentıal Equations, Dynamical Systems I, Springer-Verlag (1988).

[7] PALKA, B.P., An Introduction to Complex Function Theory, Springer-Verlag (1991).

[8] GUAN, K Y., Bifurcation of the periodic differential equations with regularisable infinity, Proc. Roy. Soc. Edin., 107A (1987), 233-247.

[9] BEARDON, A.F., The Geometry of Discrete Groups, Springer-Verlag (1983).

[10] GUAN, K.Y. and CHENG, R.Y, On the global first integral of the second order polynomial system in the complex domain and its admitted lie group, J. of Nanjing University, Mathematical Biquarterly, Nanjing (1993), 229-235 


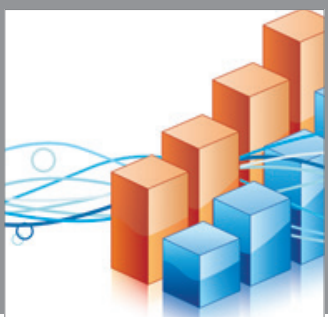

Advances in

Operations Research

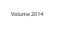

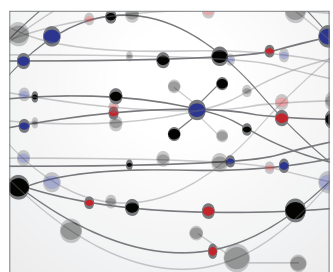

\section{The Scientific} World Journal
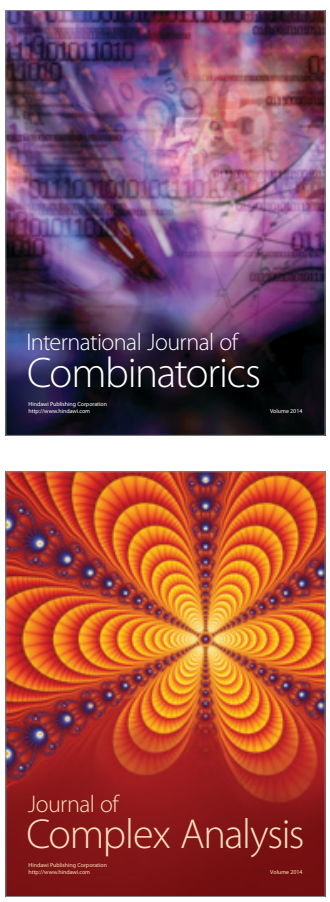

International Journal of

Mathematics and

Mathematical

Sciences
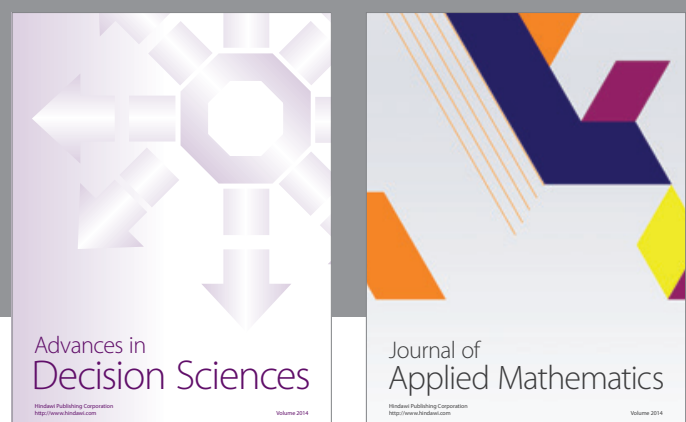

Journal of

Applied Mathematics
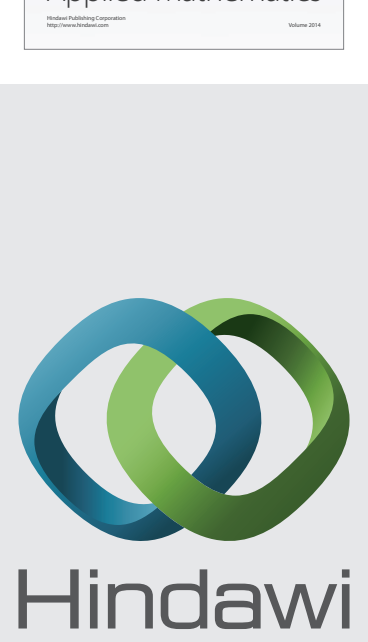

Submit your manuscripts at http://www.hindawi.com
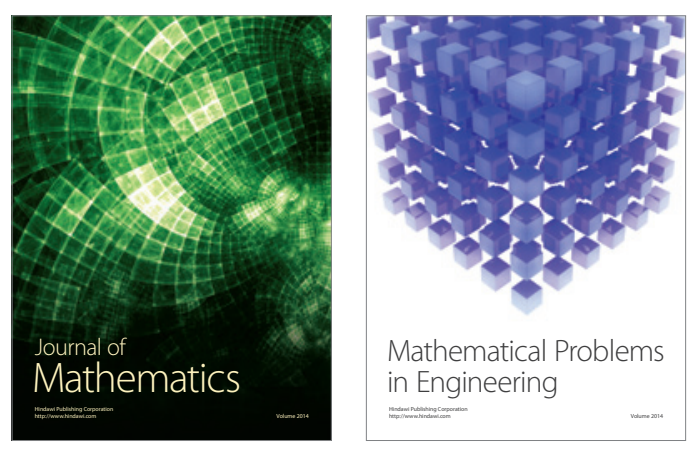

Mathematical Problems in Engineering
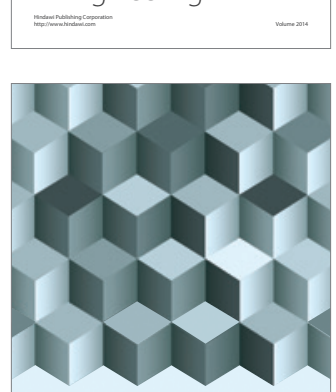

Journal of

Function Spaces
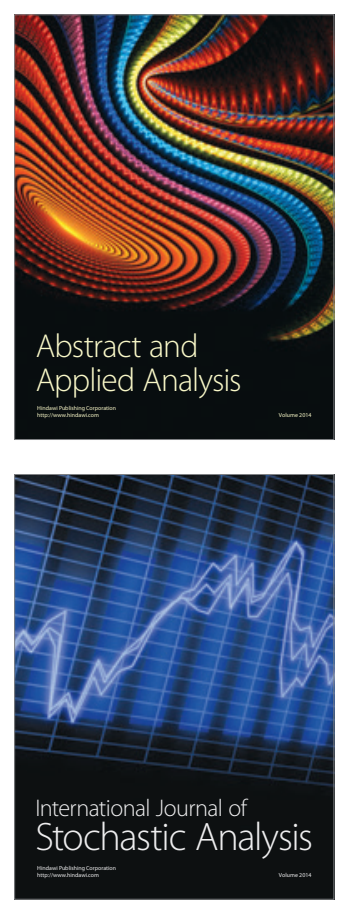

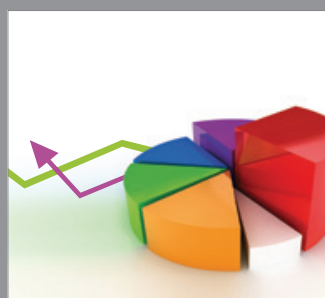

ournal of

Probability and Statistics

Promensencen
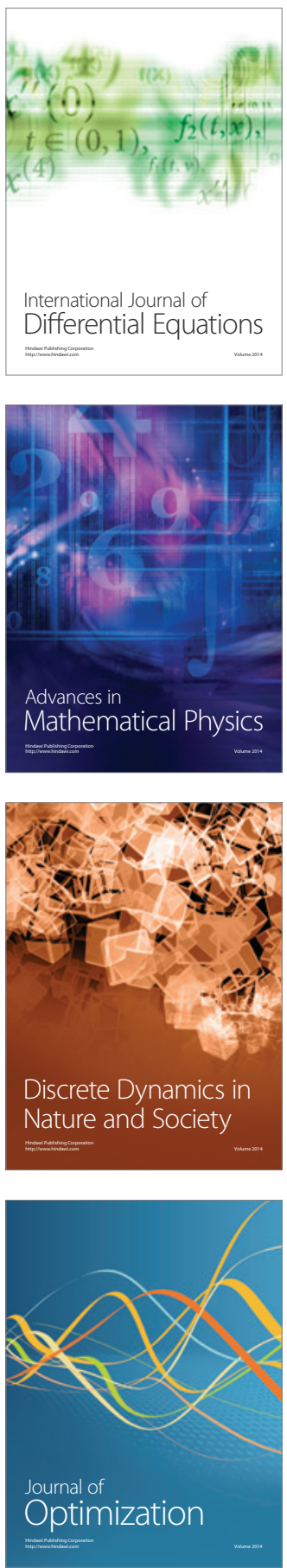